Diego Iacono, $\mathrm{MD}, \mathrm{PhD}$

Maria Geraci-Erck, BSc

Marcie L. Rabin, MD

Charles H. Adler, MD, $\mathrm{PhD}$

Geidy Serrano, PhD

Thomas G. Beach, MD, $\mathrm{PhD}$

Roger Kurlan, MD

Correspondence to

Dr. Iacono:

diego.iacono@atlantichealth.org

\title{
Parkinson disease and incidental Lewy body disease
}

\author{
Just a question of time?
}

\section{ABSTRACT}

Objective: To quantify the loss of pigmented neurons in the substantia nigra (SN) of autopsy-confirmed Parkinson disease (PD) and incidental Lewy body disease (ILBD) vs age-matched controls (C).

Methods: Unbiased stereology methods were used to rigorously count number and measure volumes of nigral pigmented neurons in PD, ILBD, and C brains. The obtained stereologic results were correlated with Lewy body (LB), amyloid plaque (AP), neurofibrillary tangle (NFT), and vascular pathology loads assessed in nigral and extranigral regions of each PD, ILBD, and C brain. The stereologic measurements were also correlated to predeath motor and cognitive scores as available for each participant.

Results: A marked nigral neuronal loss (NNL) in PD (-82\%) and ILBD (-40\%) compared to C $(p<0.0001)$ was found. While there was significant correlation between NNL and LB in some cortical areas of PD (i.e., olfactory bulb), there were no correlations between NNL and LB, AP, or NFT loads or cerebral infarct volumes in any other examined regions for PD and ILBD brains.

Conclusions: Using unbiased stereology methods, we show that there is a significant loss and absence of hypertrophic changes in nigral pigmented neurons of ILBD in comparison to $\mathrm{C}$ brains. Intriguingly, no significant correlations were found between NNL and LB loads in the SN of both PD and ILBD brains. These autopsy-verified stereologically based findings are novel and support ILBD as a pathologic condition. These results suggest possible new and alternative pathophysiologic hypotheses on the actual relationship between NNL and LB pathology. Neurology ${ }^{\circledR}$ 2015;85:1670-1679

\section{GLOSSARY}

$\mathbf{A D}=$ Alzheimer disease; $\mathbf{A P}=$ amyloid plaques; $\mathbf{B R I n j}=$ Biomedical Research Institute of New Jersey; $\mathbf{B W}=$ brain weight; $\mathbf{C}=$ controls; $\mathbf{C A A}=$ cerebral amyloid angiopathy; $\mathbf{C V}=$ cresyl violet; $\mathbf{D L B}=$ dementia with Lewy bodies; $\mathbf{H} \& \mathbf{Y}=$ Hoehn \& Yahr; ILBD = incidental Lewy body disease; LB = Lewy bodies; $\mathbf{L C}=$ locus ceruleus; $\mathbf{L T S}=$ Lewy-type synucleinopathy; MMSE = Mini-Mental State Examination; NFT = neurofibrillary tangles; NIA = National Institute on Aging; NNL = nigral neuronal loss; PD = Parkinson disease; $\mathbf{P M I}=$ postmortem interval; $\mathbf{S N}=$ substantia nigra; UPDRS = Unified Parkinson's Disease Rating Scale.

The loss of pigmented neurons in the substantia nigra ( $\mathrm{SN})$, also known as nigral neuronal loss (NNL), is one of the main pathologic features of Parkinson disease (PD). ${ }^{1,2}$ However, NNL is also observed in dementia with Lewy bodies (DLB), ${ }^{3}$ in other neurodegenerative disorders, ${ }^{4}$ and in clinically normal elders. ${ }^{5}$

Another pathologic feature of PD/DLB is the presence of Lewy bodies (LB) and Lewy neurites/aggregates, referred to as Lewy-type synucleinopathy (LTS). ${ }^{6-8}$ LTS is found in different regions of central, peripheral, and autonomic nervous systems, ${ }^{9-12}$ in extracerebral organs, ${ }^{13}$ and in clinically normal elders at autopsy. ${ }^{14}$ This is termed incidental LB disease (ILBD). ${ }^{15}$

Whether ILBD is in the preclinical PD/DLB phase or is instead resistant to LTS and the disease will not develop is not established. ${ }^{16-18}$ ILBD as a pathologic condition comes from studies describing an approximately $50 \%$ reduction in the concentrations of striatal dopaminergic markers. ${ }^{19}$ A critical deficiency, however, has been the lack of unambiguous measurement of NNL in ILBD. ${ }^{20,21}$
Supplemental data at Neurology.org
From Neuropathology Research (D.I., M.G.-E.), Biomedical Research Institute of New Jersey, BRInj, Cedar Knolls; Movement Disorders Program (D.I., M.L.R., R.K.), Atlantic Neuroscience Institute, Overlook Medical Center, Summit, NJ; Department of Neurology (D.I., R.K.), Icahn School of Medicine at Mount Sinai, Mount Sinai Hospital, New York, NY; Parkinson's Disease and Movement Disorders Center (C.H.A.), Mayo Clinic Arizona, Scottsdale; and Civin Laboratory for Neuropathology (G.S., T.G.B.), Banner Sun Health Research Institute, Sun City, AZ. Go to Neurology.org for full disclosures. Funding information and disclosures deemed relevant by the authors, if any, are provided at the end of the article. 
We aimed to (1) count the number of nigral pigmented neurons in $\mathrm{PD}$, ILBD, and controls (C) using unbiased stereology methods; (2) correlate NNL with nigral and extranigral amounts of LB, amyloid plaques (AP), neurofibrillary tangles (NFT), and motor and cognitive abnormalities before death; (3) look for possible mechanisms of cellular compensation in ILBD, i.e., increased cellular/subcellular volumes in nigral pigmented neurons of ILBD vs C; (4) test whether possible nigral neuronal volumetric changes could correlate with NNL, nigral and extranigral amounts of LB, anatomical distribution of LB, non-LTS copathologies, and motor and cognitive scores prior to death.

METHODS Participants enrolled from 1997 to 2013 in an ongoing longitudinal clinical-neuropathologic study, the Arizona Study of Aging and Neurodegenerative Disorders, with autopsies performed by the Banner Sun Health Research Institute Brain and Body Donation Program (www.brainandbodydonationprogram.org), were included. ${ }^{22}$ Participants received annual movement disorder and cognitive assessments from time of entry to time of death, at which time an autopsy was performed. Three groups of participants were defined:

1. PD: autopsy-confirmed clinical diagnosis of idiopathic PD that included at least 2 of the 3 cardinal clinical signs of PD (resting tremor, muscular rigidity, bradykinesia) and brain positivity to LTS.

2. ILBD: autopsy-confirmed brain positivity to LTS without diagnosis of PD, DLB, or other neurodegenerative disease.

3. C: no brain positivity to LTS without diagnosis of PD, DLB, or other neurodegenerative disease.

A total of 18 brains were obtained, 6 per group: $6 \mathrm{PD}, 6$ ILBD, and $6 \mathrm{C}$.

Standard protocol approvals, registrations, and patient consents. All participants signed a written informed consent approved by the Banner Sun Health Research Institute Institutional Review Board to use their medical data and donated body specimens for research purposes.

Clinical and pathologic data. For each participant, the following clinical and pathologic diagnoses were excluded: Alzheimer disease (AD), vascular dementia, DLB, progressive supranuclear palsy, corticobasal degeneration, multiple system atrophy, hippocampal sclerosis, motor neuron disease, Pick disease, Huntington disease, and frontotemporal lobar dementia with TDP-43 proteinopathy. Data on clinical and pathologic diagnoses, age at death, sex, race, cause of death, postmortem interval (PMI), brain weight (BW), and $A P O E$ genotype were available.

For each examined case, LB, AP, and NFT densities were available. LB densities (phosphorylated $\alpha$-synuclein immunohistochemistry) were measured in $\mathrm{SN}$, locus ceruleus (LC), olfactory bulb, brainstem at level of the IX and X cranial nerve, amygdala, and transentorhinal, cingulate, temporal, parietal, and frontal cortex. AP and NFT densities were assessed in frontal, temporal, parietal, and entorhinal cortex, and CA1 region of the hippocampus. The staging for each type of pathology was performed according to the Unified Staging System for Lewy
Body Disorders (for LTS), ${ }^{7}$ Consortium to Establish a Registry for Alzheimer's Disease criteria (for AP), ${ }^{23}$ and NFT-Braak system (for NFT). ${ }^{24}$ Data on depigmentation of $\mathrm{SN},{ }^{25}$ vascular pathologies, infarct volumes, and cerebral amyloid angiopathy (CAA) were also available.

As documentation for the motor and cognitive status before death, the following data were available: Unified Parkinson's Disease Rating Scale (UPDRS)-motor scale (off medication) ${ }^{26}$ Hoehn \& Yahr (H\&Y) stage, ${ }^{27}$ time interval between last UPDRS-motor scale and death, time interval between last Mini-Mental State Examination (MMSE) ${ }^{28}$ and death, cognitive impairment assessment (i.e., cognitively normal, mild cognitive impairment, or dementia), and levels of probability that dementia, if present, was due to $\mathrm{AD}$ pathology, as based on National Institute on Aging (NIA)-Reagan criteria. ${ }^{29}$

Neuropathologic material and stereology methods. Eighteen sets of 40- $\mu$ m-thick serially cut sections ( 1 every 24 from the entire $\mathrm{SN}$ ) for each participant was received at the Neuropathology Research Labs, Biomedical Research Institute of New Jersey (BRInj). Histologic and staining procedures, stereologic protocols and measurements, and statistical analyses were performed at BRInj.

Each $40-\mu \mathrm{m}$-thick section was stained with a $1.0 \%$ solution of cresyl violet $(\mathrm{CV})$ and used for the unbiased neuronal counting and volumetric measurements. Each section was randomly coded by an investigator blinded for the clinical and pathologic diagnoses (M.G.-E.) prior to any stereologic measurements, which were performed by a second clinical and pathologic diagnoses-blinded independent investigator (D.I.). Each section included both anatomical regions of $\mathrm{SN}$ : the pars compacta and pars reticulata.

We applied the following histologic criteria for neuronal counting:

1. Inclusion of any neuron, of any size, which contained neuromelanin detected by $\mathrm{CV}$ stain, within the marked SN anatomical contours.

2. Exclusion of all non-neuronal cells within the marked anatomical SN contours, such as macrophages.

We applied the following adjunctive criteria for neuronal volumetric measurements:

1. Individuation of a well-defined non-neuromelanin-covered nucleolus for each randomly sampled nigral pigmented neuron.

2. Establishing the nucleolus as the only and constant point of spatial reference for any volumetric measurement (cell body, nucleus, and nucleolus) in each randomly sampled nigral pigmented neuron

Measurements were performed using Stereo-Investigator system, version 10.0 (MBF Bioscience, Williston, VT). The sampling grid area was $500 \times 500 \mu \mathrm{m}$, with a counting frame of $40 \times 40 \mu \mathrm{m}$, disector height of $25 \mu \mathrm{m}$, and guard zones of \pm 2 $\mu \mathrm{m}$. The total number of CV-stained nigral pigmented neurons was estimated using the Optical Fractionator probe. ${ }^{30}$ The oneside $\mathrm{SN}$ neuronal counting was doubled to obtain estimation of the total neuronal population in SN (assuming no pathologic/ cellular asymmetry between SN sides in PD, ILBD, and C).

Volumetric measurements were performed using the Nucleator probe. Cell body, nuclear, and nucleolar volumes of each nigral pigmented neuron were measured by placing 6 rays automatically centered on the nucleolus and randomly intersecting the cell membrane of each single randomly sampled pigmented neuron (figure 1). All volumetric measurements were performed employing a $100 \times$ oil-immersion objective.

Statistical analyses. Analyses of variance (ANOVAs) were performed to detect group differences for mean age at death, PMI, $\mathrm{BW}$, last MMSE and UPDRS-motor (off medication) scores, 


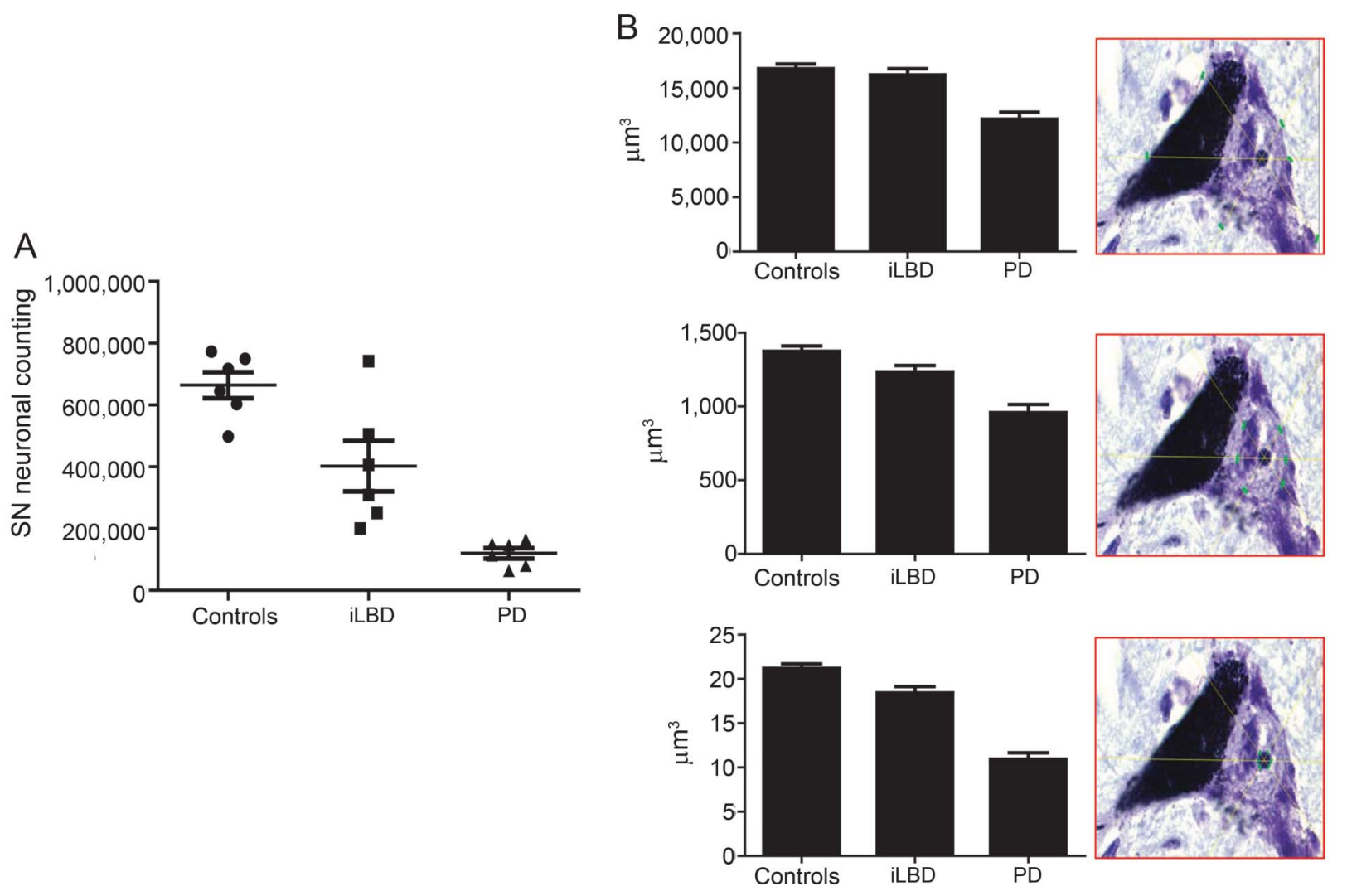

The figure shows histograms of the estimated nigral pigmented neuronal population (neuronal counting, A), and the mean cell body, nuclear, and nucleolar volumes of nigral pigmented neurons measured in PD, ILBD, and controls (B). ILBD = incidental Lewy body disease; PD = Parkinson disease; $\mathrm{SN}=$ substantia nigra.

time intervals between last MMSE/UPDRS scores and death, and $\mathrm{H} \& \mathrm{Y}$ scores.

Before statistical analyses for each type of measurement (neuronal counting, cell body, nuclear and nucleolar volume), a series of Kolmogorov-Smirnov tests were performed. Kolmogorov-Smirnov tests confirmed that all data were normally distributed. ANOVAs were followed by 2 post hoc analyses: Tukey and Bonferroni tests for multiple comparisons. Adjustments for multiple comparisons established the statistical significance at $p \leq 0.01$.

Correlation analyses were performed between neuronal counting and nigral LB, extranigral LB (LC, olfactory bulb, brainstem at level of the IX and X cranial nerves, amygdala, and transentorhinal, cingulate, temporal, parietal, and frontal cortex), AP, NFT, mean cell body, nuclear and nucleolar volume, last MMSE/ UPDRS-motor scores, and $\mathrm{H} \& \mathrm{Y}$ stages for all groups.

RESULTS Data on demographics, clinical and pathologic diagnoses, cause of death, PMI, BW, APOE genotype, motor (UPDRS-motor and $\mathrm{H} \& \mathrm{Y}$ ) scores, cognitive (MMSE, cognitive impairment, NIA-Reagan) scores, and their interval time to death are summarized in table 1. As for the mean age at death, PD and ILBD did not differ with respect to $\mathrm{C}$, while PD differed vs ILBD, with the ILBD group being older. These findings substantiated the comparability of the mean age at death between ILBD and C, and reduced possible confounding effects due to aging ${ }^{31}$ on the NNL measured in ILBD. No differences were observed for the mean PMI and BW across all 3 groups.

Table 2 summarizes the main neuropathologic findings. AP and NFT densities scores showed a substantial comparability across PD, ILBD, and C. SN depigmentation was more severe in PD compared to ILBD and C. The total brain infarct volume was higher in ILBD compared to PD and C. CAA was higher in PD vs ILBD and C.

As for the cognitive status before death and interval time between last motor/cognitive evaluation and death, no differences were found across the 3 groups. The mean last MMSE scores did not differ across groups, while UPDRS-motor scores (off medication) and modified $H \& Y$ stage were higher in PD vs ILBD and C. The PD group had 2 low cases and 1 intermediate case as for the probability of dementia due to AD pathology based on NIA-Reagan criteria. There were no statistical differences across groups for $A P O E$ allelic frequencies.

Stereologic findings. Marked NNL was found in PD and ILBD vs $C(p<0.0001)$. The mean \pm SD neuronal count was $120,015 \pm 41,051$ for PD, $402,045 \pm 199,163$ for ILBD, and 664,308 \pm 103,541 for C. In PD and ILBD, the nigral neuronal 


\begin{tabular}{|c|c|c|c|c|c|c|c|c|c|c|c|c|c|c|c|c|}
\hline \multirow{2}{*}{$\begin{array}{l}\text { Table } 1 \\
\text { ID no. }\end{array}$} & \multicolumn{16}{|c|}{$\begin{array}{l}\text { Demographic characteristics, age at death, antemortem and postmortem diagnosis, cause of death, PMI, BW, APOE genotype, cognitive status (last MMSE, cognitive impairment, dementia } \\
\text { different from AD, DLB, VaD, FTD), and motor status (last UPDRS-motor off medication, H\&Y stage) for each participant }\end{array}$} \\
\hline & $\begin{array}{l}\text { Clinical } \\
\text { diagnosis }\end{array}$ & $\begin{array}{l}\text { Pathologic } \\
\text { diagnosis }\end{array}$ & $\begin{array}{l}\text { Age at } \\
\text { death, } y\end{array}$ & Sex & Race & Cause of death & PMl, h & BW, $\mathbf{g}$ & $\begin{array}{l}\text { APOE } \\
\text { genotype }\end{array}$ & Last MMSE & $\begin{array}{l}\text { Last } \\
\text { MMSE time } \\
\text { interval, mo }\end{array}$ & $\begin{array}{l}\text { Cognitively } \\
\text { impaired } \\
\text { (i.e., MCI) }\end{array}$ & $\begin{array}{l}\text { Dementia } \\
\text { (no AD, DLB, } \\
\text { VaD, FTD) }\end{array}$ & $\begin{array}{l}\text { Last } \\
\text { UPDRS time } \\
\text { interval, mo }\end{array}$ & $\begin{array}{l}\text { Last UPDRS } \\
\text { motor score } \\
\text { (off med) }\end{array}$ & $\begin{array}{l}\text { Modified } \\
\text { H\&Y } \\
\text { stage }\end{array}$ \\
\hline 1 & PD & PD & 82 & $\mathrm{~F}$ & W & End-stage PD + AD & 3 & 1,085 & $3 / 4$ & 16 & 13 & No & Yes & 4 & NA & 3 \\
\hline 2 & PD & PD & 69 & M & W & End-stage PD & 2.25 & 1,380 & $3 / 4$ & 0 & 25 & No & Yes & 6 & 74 & 5 \\
\hline 3 & PD & PD & 79 & $\mathrm{~F}$ & W & $\begin{array}{l}\text { Failure to thrive, } \\
\text { dementia, PD }\end{array}$ & 3.5 & 1,200 & $3 / 4$ & 29 & 11 & Yes & No & 14 & 24 & 2 \\
\hline 4 & PD & PD & 84 & $\mathrm{~F}$ & W & End-stage PD & 2 & 1,220 & $3 / 3$ & 21 & 55 & No & Yes & 14 & NA & 4 \\
\hline 5 & PD & PD & 77 & $\mathrm{~F}$ & W & PD, COPD & 2.66 & 1,235 & $3 / 3$ & NA & NA & No & No & NA & NA & NA \\
\hline 6 & PD & PD & 83 & $\mathrm{~F}$ & W & $\begin{array}{l}\text { Cerebrovascular } \\
\text { accident, PD }\end{array}$ & 12 & 1,165 & $3 / 3$ & 22 & 30 & No & No & 15 & NA & 3 \\
\hline Mean & & & $79.0 \pm 5.5$ & $1 \mathrm{M} / 5 \mathrm{~F}$ & $5 \mathrm{~W}$ & & $4.2 \pm 3.8$ & $\begin{array}{l}1,214.1 \\
\pm 97.2\end{array}$ & $\begin{array}{l}\text { E3 (9), } \\
\text { E4 (3) }\end{array}$ & $17.6 \pm 10.8$ & $26.8 \pm 17.6$ & $\begin{array}{l}\text { Yes (1), } \\
\text { no (5) }\end{array}$ & & $10.6 \pm 5.1$ & $49.0 \pm 35.3$ & $3.4 \pm 1.1$ \\
\hline 7 & $\mathrm{C}$ & ILBD & 94 & $\mathrm{~F}$ & W & $\begin{array}{l}\text { End-stage COPD, heart } \\
\text { failure, emphysema }\end{array}$ & 2.5 & 1,150 & $3 / 3$ & NA & NA & No & No & 16 & 17 & 3 \\
\hline 8 & C & ILBD & 91 & $\mathrm{~F}$ & W & $\begin{array}{l}\text { Heart failure, } \\
\text { aspiration pneumonia }\end{array}$ & 3.33 & 1,120 & $3 / 3$ & 28 & 23 & No & No & 1 & 0 & 0 \\
\hline 9 & C & ILBD & 87 & $\mathrm{~F}$ & W & $\begin{array}{l}\text { Bronchial pneumonia- } \\
\text { bacterial, DM (type II) }\end{array}$ & 2 & 1,120 & $2 / 3$ & 30 & 19 & No & No & 19 & 12 & 1 \\
\hline 10 & C & ILBD & 84 & M & W & $\begin{array}{l}\text { Renal and respiratory } \\
\text { failure, COPD }\end{array}$ & 2.66 & 1,400 & $3 / 4$ & 26 & 25 & Yes & No & 2 & 7 & 0 \\
\hline 11 & $\mathrm{C}$ & ILBD & 83 & M & W & $\begin{array}{l}\text { Cardiac/respiratory } \\
\text { failure }\end{array}$ & 3 & 1,250 & $3 / 3$ & 30 & 1 & No & No & NA & NA & NA \\
\hline 12 & $\mathrm{C}$ & ILBD & 91 & M & W & $\begin{array}{l}\text { Heart failure, valvular } \\
\text { heart disease }\end{array}$ & 3 & 1,050 & $3 / 4$ & NA & NA & Yes & No & NA & NA & NA \\
\hline Mean & & & $88.3 \pm 4.3$ & $3 \mathrm{M} / 3 \mathrm{~F}$ & $5 \mathrm{~W}$ & & $2.7 \pm 0.4$ & $\begin{array}{l}1,181.6 \\
\pm 125.1\end{array}$ & $\begin{array}{l}\text { E2 (1), E3 } \\
\text { (9), E4 (2) }\end{array}$ & $28.5 \pm 1.9$ & $17.0 \pm 10.9$ & $\begin{array}{l}\text { Yes (2), } \\
\text { No (4) }\end{array}$ & & $9.5 \pm 8.0$ & $9.0 \pm 7.2$ & $1.0 \pm 1.4$ \\
\hline 13 & C & C & 81 & M & W & Respiratory arrest & 2.75 & 1,190 & $3 / 3$ & 25 & 40 & No & No & 13 & 6.5 & 0 \\
\hline 14 & C & C & 80 & M & W & $\begin{array}{l}\text { Metastatic sarcoma/ } \\
\text { carcinomatosis }\end{array}$ & 2.16 & 1,140 & $3 / 3$ & 30 & 4 & No & No & 15 & 3 & 0 \\
\hline 15 & $\mathrm{C}$ & C & 86 & M & W & $\begin{array}{l}\text { Lung cancer, } \\
\text { morphine overdose }\end{array}$ & 2.75 & 1,180 & $3 / 3$ & NA & NA & No & No & 32 & 2 & 0 \\
\hline 16 & C & C & 89 & M & W & Lung cancer & 2.5 & 1,440 & $3 / 4$ & 29 & 4 & No & No & 5 & 7 & 0 \\
\hline 17 & $\mathrm{C}$ & C & 91 & M & W & $\begin{array}{l}\text { Metastatic bladder } \\
\text { cancer }\end{array}$ & 1.5 & 1,440 & $3 / 3$ & 27 & 20 & No & No & 19 & 11 & 0 \\
\hline 18 & $\mathrm{C}$ & C & 87 & $\mathrm{~F}$ & W & $\begin{array}{l}\text { Heart failure, aortic } \\
\text { stenosis }\end{array}$ & 2.5 & 1,260 & $2 / 3$ & 26 & 19 & No & No & 24 & 1 & 0 \\
\hline Mean & & & $85.6 \pm 4.3$ & $5 \mathrm{M} / 1 \mathrm{~F}$ & $5 \mathrm{~W}$ & & $2.3 \pm 0.4$ & $\begin{array}{l}1,275.0 \\
\pm 133.5\end{array}$ & $\begin{array}{l}\text { E2 (2), E3 } \\
\text { (10), E4 (1) }\end{array}$ & $27.4 \pm 1.5$ & $17.4 \pm 8.9$ & $\begin{array}{l}\text { Yes (0), } \\
\text { No (6) }\end{array}$ & & $18.0 \pm 9.3$ & $5.0 \pm 3.7$ & $0 \pm 0$ \\
\hline
\end{tabular}

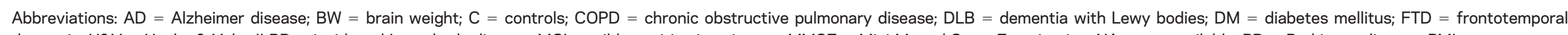

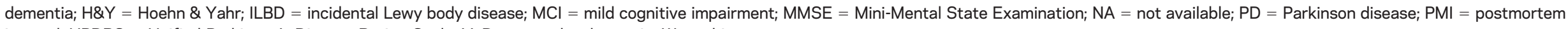
interval; UPDRS = Unified Parkinson's Disease Rating Scale; $\mathrm{VaD}=$ vascular dementia; $\mathrm{W}=$ white. 


\begin{tabular}{|c|c|c|c|c|c|c|c|c|c|c|c|c|c|c|c|}
\hline \multirow{3}{*}{$\begin{array}{l}\text { Table } 2 \\
\text { ID no. }\end{array}$} & \multicolumn{15}{|c|}{ Amyloid plaque density (CERAD), NFT Braak stages, NIA-Reagan criteria, Unified LB stage, infarct volumes, and CAA across different brain regions of each examined participant } \\
\hline & \multirow{2}{*}{$\begin{array}{l}\text { Plaque } \\
\text { density }\end{array}$} & \multirow[b]{2}{*}{$\begin{array}{l}\text { Braak } \\
\text { score }\end{array}$} & \multirow[b]{2}{*}{$\begin{array}{l}\text { NIA-Reagan } \\
\text { Criteria }\end{array}$} & \multirow[b]{2}{*}{$\begin{array}{l}\text { Unified LB } \\
\text { stage }\end{array}$} & \multirow[b]{2}{*}{$\begin{array}{l}\text { SN } \\
\text { depigmentation }\end{array}$} & \multicolumn{5}{|c|}{ Infarct volume } & \multicolumn{5}{|l|}{ CAA } \\
\hline & & & & & & Cortical & $\begin{array}{l}\text { Centrum } \\
\text { ovale }\end{array}$ & $\begin{array}{l}\text { Deep } \\
\text { nuclei }\end{array}$ & Infratentorial & Brain total & Frontal & Temporal & Parietal & Occipital & Total \\
\hline 1 & Sparse & III & Low & $\begin{array}{l}\text { Brainstem/ } \\
\text { limbic }\end{array}$ & Severe & 0 & 0 & 0 & 0 & 0 & 0 & 0 & 0 & 0 & 0 \\
\hline 2 & Sparse & ॥ & Low & Neocortical & Severe & 0 & 0 & 0 & 0 & 0 & 0 & 0 & 0 & 0 & 0 \\
\hline 3 & Moderate & IV & Intermediate & Neocortical & Severe & 0 & 0 & 0 & 0 & 0 & 1 & 1 & 1 & 2 & 5 \\
\hline 4 & Zero & ॥ & Low & Brainstem & Severe & 0 & 0 & 0 & 0 & 0 & 2 & 2 & 2 & 2 & 8 \\
\hline 5 & Zero & ॥ & Not met & Brainstem & Severe & 0 & 0 & 0 & 0 & 0 & 1 & 1 & 1 & 1 & 4 \\
\hline 6 & Sparse & III & Not met & $\begin{array}{l}\text { Brainstem/ } \\
\text { limbic }\end{array}$ & Severe & 0 & 0 & 0 & 0 & 0 & 1 & 1 & 1 & 2 & 5 \\
\hline Mean & & & & & & 0 & 0 & 0 & 0 & 0 & $0.8 \pm 0.7$ & $0.8 \pm 0.7$ & $0.8 \pm 0.7$ & $1.1 \pm 0.9$ & $5.5 \pm 1.7$ \\
\hline 7 & Moderate & III & Not met & Limbic & Moderate & 0 & 0 & 0 & 0 & 0 & 0 & 0 & 0 & 0 & 0 \\
\hline 8 & Sparse & III & Not met & Brainstem & None & 0.5 & 0 & 0 & 0 & 0.5 & 0 & 0 & 0 & 0 & 0 \\
\hline 9 & Moderate & III & Not met & Brainstem & Mild & 0 & 0 & 1 & 0 & 1 & 0 & 0 & 0 & 0 & 0 \\
\hline 10 & Zero & I & Not met & Brainstem & Moderate & 0 & 0 & 0 & 0 & 0 & 0 & 0 & 0 & 0 & 0 \\
\hline 11 & Sparse & III & Not met & Neocortical & None & 0 & 0 & 0 & 0.5 & 0.5 & 1 & 0 & 0 & 2 & 2 \\
\hline 12 & Moderate & III & Not met & Brainstem & Severe & 0 & 2 & 0 & 0.1 & 2.1 & 0 & 0 & 0 & 2 & 2 \\
\hline Mean & & & & & & $0.08 \pm 0.2$ & $0.3 \pm 0.8$ & $0.1 \pm 0.4$ & $0.1 \pm 0.2$ & $0.6 \pm 0.7$ & $0.1 \pm 0.4$ & 0 & 0 & $0.6 \pm 1.0$ & $0.6 \pm 1.0$ \\
\hline 13 & Sparse & III & Not met & No LBs & None & 0 & 0 & 0 & 0 & 0 & 0 & 1 & 1 & 0 & 2 \\
\hline 14 & Zero & I & Not met & No LBs & None & 0 & 0 & 0 & 0 & 0 & 0 & 0 & 0 & 0 & 0 \\
\hline 15 & Moderate & ॥ & Not met & No LBs & None & 0 & 0 & 0 & 0 & 0 & 0 & 0 & 0 & 0 & 0 \\
\hline 16 & Moderate & ॥ & Not met & No LBs & None & 0 & 0 & 0 & 0 & 0 & 0 & 0 & 0 & 0 & 0 \\
\hline 17 & Zero & ॥ & Not met & No LBs & None & 0 & 0 & 0 & 0 & 0 & 0 & 0 & 0 & 1 & 1 \\
\hline 18 & Moderate & III & Not met & No LBs & Mild & 0 & 0 & 0 & 0 & 0 & 0 & 0 & 0 & 0 & 0 \\
\hline Mean & & & & & & 0 & 0 & 0 & 0 & 0 & 0 & $0.1 \pm 0.4$ & $0.1 \pm 0.4$ & $0.1 \pm 0.4$ & $0.5 \pm 0.8$ \\
\hline
\end{tabular}

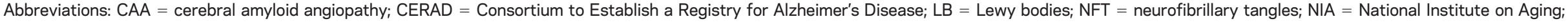
$\mathrm{SN}=$ substantia nigra. 


\begin{tabular}{|c|c|c|c|c|c|c|c|c|c|c|c|}
\hline \multirow[t]{3}{*}{ Table 3} & \multicolumn{11}{|c|}{$\begin{array}{l}\text { Single correlation values }(r= \pm \text { ) for (upper part) each type of correlation between neuronal counting, mean cell body, nuclear, and nucleolar volume and LB scores obtained in the SN, LC, } \\
\text { olfactory bulb, IX and X cranial nerve, amygdala, and transentorhinal, cingulate, temporal, parietal, and frontal cortex of PD and ILBD; and (lower part) each type of correlation between } \\
\text { neuronal counting, mean cell body, nuclear, and nucleolar volume and AP, and NFT loads, obtained in the hippocampus, entorhinal, temporal, parietal, and frontal cortex, of PD and ILBD }\end{array}$} \\
\hline & & \multicolumn{10}{|l|}{ LB } \\
\hline & & SN & LC & Olfactory bulb & $\mathrm{IX}, \mathrm{X}$ cranial nerve & Amygdala & Transentorhinal & Cingulate & Temporal & Parietal & Frontal \\
\hline \multicolumn{12}{|l|}{ ILBD } \\
\hline \multicolumn{2}{|c|}{ Neuronal counting } & 0.4712, no & -0.3286, no & 0.04422, no & -0.3019, no & -0.4189, no & -0.5442, no & -0.07312, no & -0.4790, no & -0.4956, no & -0.4790, no \\
\hline \multicolumn{2}{|c|}{ Cell body volume } & -0.02956, no & -0.5051, no & -0.1232, no & -0.4481 , no & -0.8442, yes $^{a}$ & -0.6908, no & -0.5153, no & -0.6826, no & -0.7982, no & -0.6826, no \\
\hline \multicolumn{2}{|c|}{ Nuclear volume } & 0.07948, no & -0.4767, no & -0.09271, no & -0.4126, no & -0.7960, no & -0.6735, no & -0.4516, no & -0.6867, no & -0.7806, no & -0.6867, no \\
\hline \multicolumn{2}{|c|}{ Nucleolar volume } & 0.07239, no & -0.6736, no & 0.04505, no & -0.6729, no & -0.6413, no & -0.8560, yes $^{a}$ & -0.2781, no & -0.3963, no & -0.5459, no & -0.3963, no \\
\hline \multicolumn{12}{|l|}{ PD } \\
\hline \multicolumn{2}{|c|}{ Neuronal counting } & 0.2675, no & 0.3922, no & -0.8864, yes $^{a}$ & -0.4682 , no & -0.2898, no & -0.4883, no & $-0.8733, y^{2} s^{a}$ & -0.8356, yes $^{a}$ & -0.8515, yes $^{a}$ & -0.6420, no \\
\hline \multicolumn{2}{|c|}{ Cell body volume } & 0.4089 , no & 0.4518, no & -0.7396, no & -0.7485, no & -0.6702, no & -0.8325, yes $^{a}$ & -0.9025, yes $^{a}$ & -0.9407, yes $^{a}$ & -0.6246, no & -0.6592, no \\
\hline \multicolumn{2}{|c|}{ Nuclear volume } & 0.6554, no & -0.3074, no & 0.1774, no & -0.4254, no & -0.7547, no & -0.6126, no & -0.1816, no & -0.2603, no & 0.03238, no & -0.07885, no \\
\hline \multirow{3}{*}{\multicolumn{2}{|c|}{ Nucleolar volume }} & 0.5785, no & -0.4328, no & 0.3782, no & 0.5634, no & 0.01099, no & 0.4382 , no & 0.3271, no & 0.2921, no & 0.08627, no & 0.1688, no \\
\hline & & AP & & & & & NFT & & & & \\
\hline & & Hippocampus & Entorhinal & Temporal & Parietal & Frontal & Hippocampus & Entorhinal & Temporal & Parietal & Frontal \\
\hline \multicolumn{12}{|l|}{ ILBD } \\
\hline \multicolumn{2}{|c|}{ Neuronal counting } & -0.2301, no & -0.3865, no & -0.5380, no & -0.3099, no & -0.3099, no & -0.3756, no & -0.3756, no & -0.4583, no & -0.4956, no & -0.4956, no \\
\hline \multicolumn{2}{|c|}{ Cell body volume } & 0.3665, no & 0.006908, no & 0.1514, no & 0.3467, no & 0.3467, no & 0.2251, no & 0.1523, no & -0.6949, no & -0.7982, no & -0.7982, no \\
\hline \multicolumn{2}{|c|}{ Nuclear volume } & 0.1984, no & -0.07011, no & -0.04454, no & 0.2014, no & 0.2014, no & 0.05429, no & 0.01320, no & -0.6383, no & -0.7806, no & -0.7806, no \\
\hline \multicolumn{2}{|c|}{ Nucleolar volume } & 0.5485, no & -0.2032, no & 0.3492, no & 0.2860, no & 0.2860, no & 0.5189, no & 0.2270, no & -0.8081, no & -0.5459, no & -0.5459, no \\
\hline \multicolumn{12}{|c|}{ PD } \\
\hline \multicolumn{2}{|c|}{ Neuronal counting } & -0.6881 , no & -0.5755, no & -0.5121, no & -0.4543, no & -0.7313, no & -0.1388, no & -0.2874, no & 0.1336, no & -0.6701, no & -0.6701, no \\
\hline \multicolumn{2}{|c|}{ Cell body volume } & -0.6442, no & -0.5442, no & -0.3661, no & -0.4478, no & -0.8342, yes $^{a}$ & -0.4366, no & -0.5153, no & 0.2323, no & -0.5233, no & -0.5233, no \\
\hline \multicolumn{2}{|c|}{ Nuclear volume } & 0.1468, no & 0.2474, no & 0.3155, no & 0.2993, no & 0.3732, no & -0.1831, no & -0.3636, no & -0.2855, no & -0.3188, no & -0.3188, no \\
\hline \multicolumn{2}{|c|}{ Nucleolar volume } & 0.3482, no & 0.5505, no & 0.3384, no & -0.01511, no & 0.3056 , no & 0.05003, no & -0.2977, no & -0.6540, no & 0.2966, no & 0.2966 , no \\
\hline
\end{tabular}

Abbreviations: AP = amyloid plaques; ILBD = incidental Lewy body disease; LB = Lewy bodies; LC = locus ceruleus; NFT = neurofibrillary tangles; PD = Parkinson disease; SN = substantia nigra. Yes = significant linear correlation; no = no significant linear correlation.

a Significant correlation. 
counting was, respectively, $82 \%$ and $40 \%$ lower than $\mathrm{C}$ (figure 1).

ANOVAs did not show differences for the mean nigral cell body volume between ILBD and C. Moreover, while the mean cell body, nuclear, and nucleolar volumes were lower (as for a possible subjacent atrophic process) in PD vs ILBD and C ( $p<0.0001)$, the mean nuclear and nucleolar volumes were lower in both PD and ILBD vs $C(p<0.0001)$. These quantitative volumetric measurements seem to exclude cellular compensatory phenomena, for example a neuronal hypertrophy, in nigral pigmented neurons of ILBD in comparison to $\mathrm{C}$.

The PD group showed correlations between NNL and LB in extranigral regions of the brain, such as olfactory bulb, cingulate, temporal, and parietal cortex. In the ILBD group, by contrast, no correlations were found between NNL and LB in any examined brain region. Furthermore, in both PD and ILBD groups, there were no correlations between NNL and AP or NFT densities, infarct volumes, and CAA.

In the ILBD group, correlations between mean cell body/nucleolar volume and LTS were found in amygdala and transentorhinal cortex, while in the PD group those correlations were found only in transentorhinal, cingulate, and temporal cortex.

No correlations were found in the ILBD or PD group between NNL and neuronal volumes, AP, and NFT densities; the only exception was found in the PD group, where the mean cell body volume and AP densities were correlated in the frontal cortex. No correlations were found between NNL and last UPDRS-motor scores and H\&Y stages in either PD or ILBD.

Table 3 shows the single correlation values (Pearson $r$ ) among neuronal counting, mean cell body, nuclear, and nucleolar volume with LB, AP, and NFT densities for each assessed brain region.

Figure 1 shows histograms for the estimated neuronal counting, mean cell body, nuclear, and nucleolar volumes of nigral pigmented neurons in $\mathrm{PD}$, ILBD, and C. Figure 2 shows graphic relationships between NNL and their cell body volume, as between NNL and the LB sum densities in PD, ILBD, and C. Table e-1 on the Neurology ${ }^{\circledR}$ Web site at Neurology.org, upper part, using a color-based coding system, describes the neuroanatomical distribution of LB pathology and its level of severity (none, mild, moderate, severe, very severe) across all groups. Table e-1, lower part, using a color-based coding system as well, describes the presence (yes) or the absence (no) of LB across all examined cerebral regions.

DISCUSSION Whether ILBD participants represent individuals who did not live long enough to develop PD or DLB, or instead they represent a condition of asymptomaticity and neuronal resistance to LTS and NNL, are 2 important pathophysiologic questions whose answers could imply, potentially, new ways on how to prevent, halt, or at least delay the progression of ILBD in PD or DLB. So far, it is not possible to definitely establish if PD and DLB are manifestations of different pathogenetic processes or if they are expressions of an identical pathologic process

Figure 2 Nigral pigmented neurons related to cellular volumes and LB densities in PD, ILBD, and controls

A

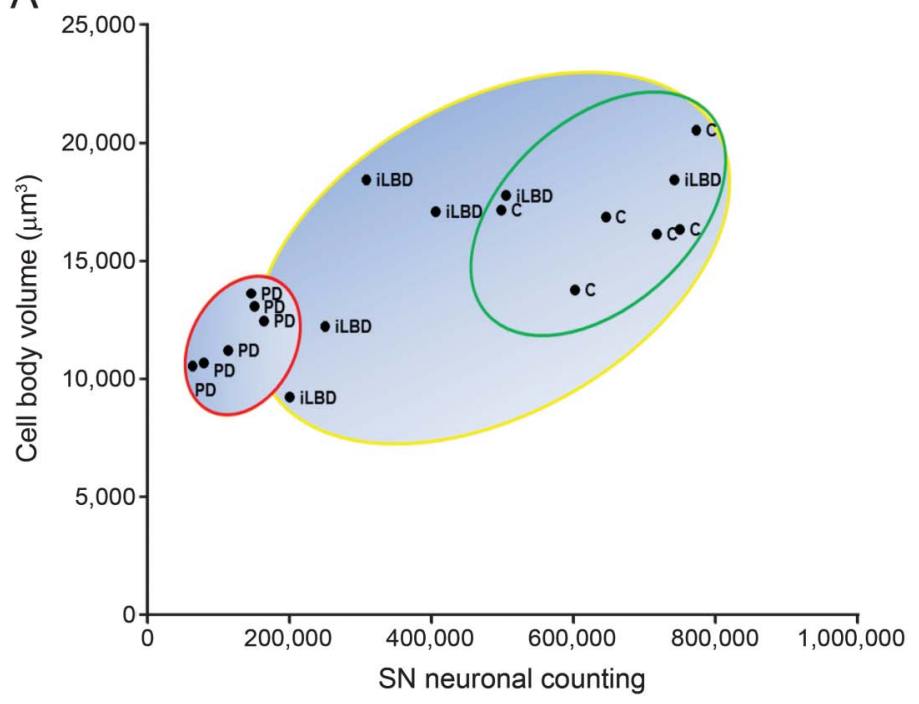

B

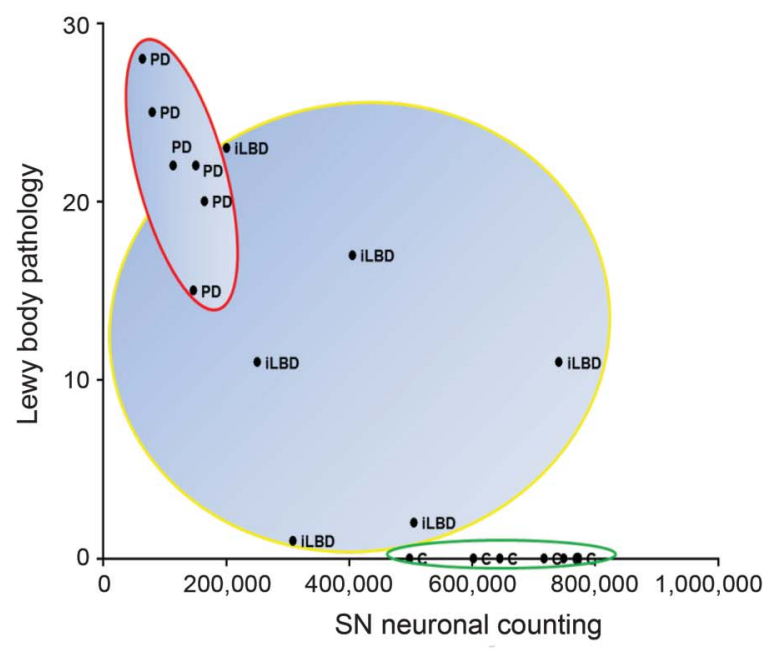

The figure shows, graphically, the correlations between mean cell body volume and nigral pigmented neuronal counting (A), and between Lewy body pathology (sum of the Lewy body density across all examined regions) and nigral pigmented neuronal counting (B) in PD, ILBD, and C participants. C = control; ILBD = incidental Lewy body disease; PD = Parkinson disease; $\mathrm{SN}=$ substantia nigra. 
related to the specific characteristics of neuronal vulnerability in different participants, to possible compensatory mechanisms, or to peculiar anatomical characteristics of the neuronal circuits involved in the initial formation and spreading of LTS. ${ }^{8,32}$ Since both PD and DLB are diseases pathologically characterized by nigral accumulation of LB/LTS, and both could have ILBD as a preclinical phase, it was of crucial importance to establish whether there is an unequivocal NNL in ILBD, and if so, which could be its severity in comparison to PD and age-matched C. Our quantitative stereologic findings show NNL in ILBD participants in comparison to age-matched control elders. In addition, although we found correlations between some extranigral LB burdens and NNL, no correlation was found within the SN. One of the possible explanations for this may be that the high rate of cell death and subsequent phagocytosis of LB/LTS could obscure the direct relationship between NNL and LB/LTS. A more drastic hypothesis could imply that LB/LTS is not related to NNL at all and that the observed NNL could just be an aging-related phenomenon coexisting with a simultaneous independent process of LB/LTS formation and accumulation. This latter pathologic process could be induced by triggers that are different from those causing NNL.

A further hypothesis could be that the formation of LB/LTS begins, or is inducible, only in some specific and more vulnerable extranigral cerebral areas (i.e., olfactory bulb? Nucleus motor dorsal vagus? Others?) from which LB/LTS pathology can be spread across multiple extranigral regions of the brain as well as to the SN. This hypothesis could explain the appearance of initial dystrophic/atrophic phenomena and consequent NNL as a progressive spectrum of degenerative cellular phenomena related to the synucleinopathic process and its progression. This hypothesis, moreover, fits well with the prion-like theory recently proposed to better explain the spreading of $\alpha$-synucleinopathies across preferential neuronal circuits and anatomical directions. ${ }^{33}$

Our findings show that in the nigral pigmented neurons of ILBD brains, subcellular abnormalities, such as nuclear and nucleolar decreased volumes (possible signals of neuronal atrophy), are indeed present in the context of an already significant NNL, and importantly, in the absence of a clinically relevant motor and cognitive symptomatology. This supports the hypothesis that ILBD is a preclinical phase of PD/DLB. Some previous nonstereologic investigations supported this hypothesis. ${ }^{34}$ However, those studies ${ }^{34}$ could not detect a significant NNL in ILBD compared to controls. Here, using unbiased stereology methods, we show that a significant neuronal loss is actually already present in the SN of ILBD in comparison to C.

Our measurements show that a $40 \%$ reduction of NNL in ILBD vs $\mathrm{C}$ is still compatible with an absence of clinically significant motor or cognitive symptoms. This suggests that although some resistive or compensatory mechanisms may not be present at cellular level, inside or outside the $\mathrm{SN}$, they could actually be activated at neurotrasmettitorial, receptorial, synaptic, or even at molecular level.

Further, it could be hypothesized that the total number of pigmented neurons in the $\mathrm{SN}$ of a normally developed adult brain could actually exceed the minimal number of neurons required for a normal functioning of SN. This "nigral neuronal reserve" could explain why, for example, in clinically normal and LTS-negative elders the presence of NNL is not always or mandatorily associated with clinically relevant motor or cognitive symptoms.

PD and ILBD, however, and although with different amounts, often have overlapping LTS cerebral distributions (table 2 and table e-1). So important questions remain open: How might ILBD evolve, if it evolves, towards $\mathrm{PD}$ or DLB? Is this just a question of time? If ILBD represents a preclinical phase subjacent to an identical pathogenetic process ( $\alpha$-synucleinopathy) for both PD and DLB, is there a different neuronal vulnerability? Possible compensatory or different vulnerability properties, as well as protective factors, should be taken into account since aging per se does not seem to play a major additive role in causing NNL in ILBD. In this study, the ILBD group was older than the PD group, and previous studies show comparable results. ${ }^{9,20}$ It is also important to consider that specific genotypes (i.e., $L R R K 2$ gene and its variants) as well as environmental cofactors could accelerate or decelerate the progression/deposition of LB and consequent NNL. ${ }^{35}$

Our study presents some caveats: the relatively small sample size, the unavailability of quantitative measurements (i.e., using stereologic tools) of the various brain copathologies (LTS, AP, NFT) present in those individuals, and positive UPDRS scores in some ILBD and C. Although positive UPDRS scores in older participants are not rarely observed due to various medical conditions, more sophisticated clinical assessments should be performed to exclude more subtle extrapyramidal signs.

Larger autopsy quantitative stereology-based studies need to confirm our novel findings. We support that ILBD is a genuine pathologic status, and that it can represent a preclinical phase of $\mathrm{PD} / \mathrm{DLB}$, since we found significant NNL in those brains in comparison to C. Furthermore, the lack of hypertrophy in nigral pigmented neurons does not support the presence of cellular compensatory mechanisms in the $\mathrm{SN}$ of ILBD brains. Moreover, cortical AP and NFT densities, as infarct volumes and CAA, do not seem to have a major contributing role in determining NNL. 
Alternative hypotheses remain open:

1. ILBD is not the preclinical phase of PD/DLB per se, but an anatomically parallel and pathogenetically separated process overlapping other pathologic events causing PD/DLB. ${ }^{35}$ Those PD/DLB-specific pathologic events could simply enhance degenerative phenomena in already predisposed or particularly vulnerable brain areas. An intriguing similitude here could be evoked with the recently defined condition of primary age-related tauopathy. ${ }^{36}$ Could ILBD be a condition of primary age-related synucleinopathy? Is it just by chance that the olfactory dysfunction normally present during aging and ILBD is accentuated in PD? ${ }^{37}$

2. ILBD is indeed a preclinical phase of PD/DLB but it requires a longer gestation period to be clinically manifested in some predisposed participants. ${ }^{38}$

3. ILBD is not a preclinical phase at all, despite a $40 \%$ NNL vs C, but simply represents a status of asymptomaticity, perhaps lasting until death and based on unknown protective factors ${ }^{39,40} \mathrm{lim}$ iting the progression of NNL.

\section{AUTHOR CONTRIBUTIONS}

Dr. Iacono: study concept and design. M. Geraci-Erck: acquisition data and technical support. Dr. Rabin: critical revision of the manuscript. Dr. Adler: critical revision of the manuscript. Dr. Serrano: acquisition data and technical support. Dr. Beach: critical revision of the manuscript. Dr. Kurlan: critical revision of the manuscript.

\section{STUDY FUNDING}

Supported by the Biomedical Research Institute of New Jersey (BRInj), through the PD and Other Movement Disorders Research Grant 2014.

\section{DISCLOSURE}

D. Iacono and M. Geraci-Erck report no disclosures relevant to the manuscript. M. Rabin reports personal fees from Teva, Allergan, and Ipsen. C. Adler reports personal fees from Lilly, Merz, Lundbeck, Teva, Acadia, and AbbVie. G. Serrano reports no disclosures relevant to the manuscript. T. Beach reports grants from the US National Institute for Neurological Disease and Stroke, US National Institute on Aging, Michael J. Fox Foundation for Parkinson's Research, State of Arizona, and Avid Radiopharmaceuticals, and personal fees from GE Healthcare. R. Kurlan reports grants from NIH, Astra-Zeneca, Psyadon, Rhythm, Kyowa, and Teva. Go to Neurology.org for full disclosures.

Received February 10, 2015. Accepted in final form July 7, 2015.

\section{REFERENCES}

1. Jellinger KA. Neuropathology of sporadic Parkinson's disease: evaluation and changes of concepts. Mov Disord 2012;27:8-30.

2. Lees AJ, Hardy J, Revesz T. Parkinson's disease. Lancet 2009;373:2055-2066.

3. Seidel K, Mahlke J, Siswanto S, et al. The brainstem pathologies of Parkinson's disease and dementia with Lewy bodies. Brain Pathol 2015;25:121-135.

4. Oyanagi K, Tsuchiya K, Yamazaki M, et al. Substantia nigra in progressive supranuclear palsy, corticobasal degeneration, and parkinsonism-dementia complex of Guam: specific pathological features. J Neuropathol Exp Neurol 2001;60:393-402.

5. Buchman AS, Shulman JM, Nag S, et al. Nigral pathology and parkinsonian signs in elders without Parkinson disease. Ann Neurol 2012;71:258-266.

6. Spillantini MG, Schmidt ML, Lee VM, et al. Alpha-synuclein in Lewy bodies. Nature 1997;388:839-840.

7. Beach TG, Adler CH, Lue L, et al; Arizona Parkinson's Disease Consortium. Unified staging system for Lewy body disorders: correlation with nigrostriatal degeneration, cognitive impairment and motor dysfunction. Acta Neuropathol 2009; 117:613-634.

8. Halliday GM, McCann H. Human-based studies on alpha-synuclein deposition and relationship to Parkinson's disease symptoms. Exp Neurol 2008;209:12-21.

9. Beach TG, Adler CH, Sue LI, et al. Multi-organ distribution of phosphorylated alpha-synuclein histopathology in subjects with Lewy body disorders. Acta Neuropathol 2010;119:689-702.

10. Wakabayashi K, Takahashi H. Neuropathology of autonomic nervous system in Parkinson's disease. Eur Neurol 1997;38(suppl 2):2-7.

11. Shannon KM, Keshavarzian A, Mutlu E, et al. Alpha-synuclein in colonic submucosa in early untreated Parkinson's disease. Mov Disord 2012;27:709-715.

12. Bloch A, Probst A, Bissig H, et al. Alpha-synuclein pathology of the spinal and peripheral autonomic nervous system in neurologically unimpaired elderly subjects. Neuropathol Appl Neurobiol 2006;32:284-295.

13. Gelpi E, Navarro-Otano J, Tolosa E, et al. Multiple organ involvement by alpha-synuclein pathology in Lewy body disorders. Mov Disord 2014;29:1010-1018.

14. Ben-Shlomo Y, Wenning G. Incidental Lewy body disease. Lancet 1994;344:1503.

15. Adler CH, Connor DJ, Hentz JG, et al. Incidental Lewy body disease: clinical comparison to a control cohort. Mov Disord 2010;25:642-646.

16. Frigerio R, Fujishiro H, Ahn TB, et al. Incidental Lewy body disease: do some cases represent a preclinical stage of dementia with Lewy bodies? Neurobiol Aging 2011;32:857-863.

17. DelleDonne $\mathrm{A}$, Klos $\mathrm{KJ}$, Fujishiro $\mathrm{H}$, et al. Incidental Lewy body disease and preclinical Parkinson disease. Arch Neurol 2008;65:1074-1080.

18. Jellinger KA. Lewy body-related alpha-synucleinopathy in the aged human brain. J Neural Transm 2004;111:1219-1235.

19. Beach TG, Adler CH, Sue LI, et al. Reduced striatal tyrosine hydroxylase in incidental Lewy body disease. Acta Neuropathol 2008;115:445-451.

20. Milber JM, Noorigian JV, Morley JF, et al. Lewy pathology is not the first sign of degeneration in vulnerable neurons in Parkinson disease. Neurology 2012;79:2307-2314.

21. Ross GW, Petrovitch H, Abbott RD, et al. Parkinsonian signs and substantia nigra neuron density in descendants elders without PD. Ann Neurol 2004;56:532-539.

22. Beach TG, Sue LI, Walker DG, et al. The sun health research Institute brain donation program: description and experience, 1987-2007. Cell Tissue Bank 2008;9:229-245.

23. Mirra SS, Heyman A, McKeel D, et al. The Consortium to Establish a Registry for Alzheimer's Disease (CERAD): part II: standardization of the neuropathologic assessment of Alzheimer's disease. Neurology 1991;41:479-486.

24. Braak H, Braak E. Demonstration of amyloid deposits and neurofibrillary changes in whole brain sections. Brain Pathol 1991;1:213-216. 
25. Dickson DW, Braak H, Duda JE, et al. Neuropathological assessment of Parkinson's disease: refining the diagnostic criteria. Lancet Neurol 2009;8:1150-1157.

26. Fahn S, Marsden CD, Calne DB, Goldstein M, et al. Recent Developments in Parkinson's Disease, vol 2. Florham Park, NJ: Macmillan Health Care Information; 1987:153-163.

27. Hoehn MM, Yahr MD. Parkinsonism: onset, progression and mortality. Neurology 1967;17:427-442.

28. Folstein MF, Folstein SE, McHugh PR. "Mini-mental state": a practical method for grading the cognitive state of patients for the clinician. J Psychiatr Res 1975;12:189-198.

29. The National Institute on Aging, and Reagan Institute Working group on Diagnostic Criteria for the Neuropathological Assessment of Alzheimer's disease Consensus recommendations for the postmortem diagnosis of Alzheimer's disease. Neurobiol Aging 1997;18:S1-S2.

30. Gundersen HJ, Bagger P, Bendtsen TF, et al. The new stereological tools: disector, fractionator, nucleator and point sampled intercepts and their use in pathological research and diagnosis. APMIS 1988;96:857-881.

31. Rudow G, O’Brien R, Savonenko AV, et al. Morphometry of the human substantia nigra in ageing and Parkinson's disease. Acta Neuropathol 2008;115:461-470.

32. Richard IH, Papka M, Rubio A, et al. Parkinson's disease and dementia with Lewy bodies: one disease or two? Mov Disord 2002;17:1161-1165.
33. Olanow CW, Brundin P. Parkinson's disease and alpha synuclein: is Parkinson's disease a prion-like disorder? Mov Disord 2013;28:31-40.

34. Dickson DW, Fujishiro H, DelleDonne A, et al. Evidence that incidental Lewy body disease is pre-symptomatic Parkinson's disease. Acta Neuropathol 2008;115:437-444.

35. Kurlan R. Do Parkinson's disease and dementia with Lewy bodies differ by route of environmental precipitant? Mov Disord 2008;23:1617.

36. Crary JF, Trojanowski JQ, Schneider JA, et al. Primary agerelated tauopathy (PART): a common pathology associated with human aging. Acta Neuropathol 2014;128:755-766.

37. Driver-Dunckley E, Adler CH, Hentz JG, et al. Olfactory dysfunction in incidental Lewy body disease and Parkinson's disease. Parkinsonism Relat Disord 2014;20:1260-1262.

38. Clayton DF, George JM. Synucleins in synaptic plasticity and neurodegenerative disorders. J Neurosci Res 1999;58: 120-129.

39. Colosimo C, Hughes AJ, Kilford L, et al. Lewy body cortical involvement may not always predict dementia in Parkinson's disease. J Neurol Neurosurg Psychiatry 2003; 74:852-856.

40. Ferrer I, Martinez A, Blanco R, et al. Neuropathology of sporadic Parkinson disease before the appearance of parkinsonism: preclinical Parkinson disease. J Neural Transm 2011;118:821-839.

\section{WriteClick ${ }^{\circledR}$ rapid online correspondence}

Have a comment on a recent Neurology ${ }^{\circledR}$ article you would like to share? Now it is easier and more convenient. Neurology.org has launched WriteClick on the home page and sidebars of each article to encourage remarks and debate among users.

WriteClick is restricted to comments about studies published in Neurology within the last eight weeks.

Learn more at Neurology.org/letters

\section{The Best Way to Discuss Solutions Is Face-to-face}

Join the AAN for 2016 Neurology on the Hill and help educate members of Congress so we can address our health policy issues together. If selected, you will attend this highly successful program from February 29 to March 1, 2016, and receive training from advocacy and communication coaches, veteran advocates, and AAN staff who will bring you up to date on recent issues. Then, we will go to Capitol Hill for face-to-face meetings with congressional members and their staffs. The Academy will cover travel expenses and hotel accommodations. There is a general registration fee of $\$ 150$, or $\$ 50$ for residents, fellows, and members residing in the Washington, DC, area. Encourage your colleagues to become involved and apply as well. Space is limited and fills quickly. The application deadline is December 2, 2015. Learn more and apply today at $A A N$.com/view/2016NOH. 